\title{
Tarry Stool Occurred after Replacement of Percutaneous Endoscopic Gastrostomy Tube
}

\section{Kyung Ho Song}

Department of Internal Medicine, Konyang University Hospital, Daejeon, Korea

Question: A 77-year-old man came to the emergency room because he defecated tarry stool. His hemoglobin decreased by $4.5 \mathrm{~g} / \mathrm{dL}$ compared to the previous test.
Blood pressure was 90/60 $\mathrm{mmHg}$, and pulse rate was 110 per minute. The patient had right hemiplegia with a stroke 18 months ago and was taking clopidogrel.
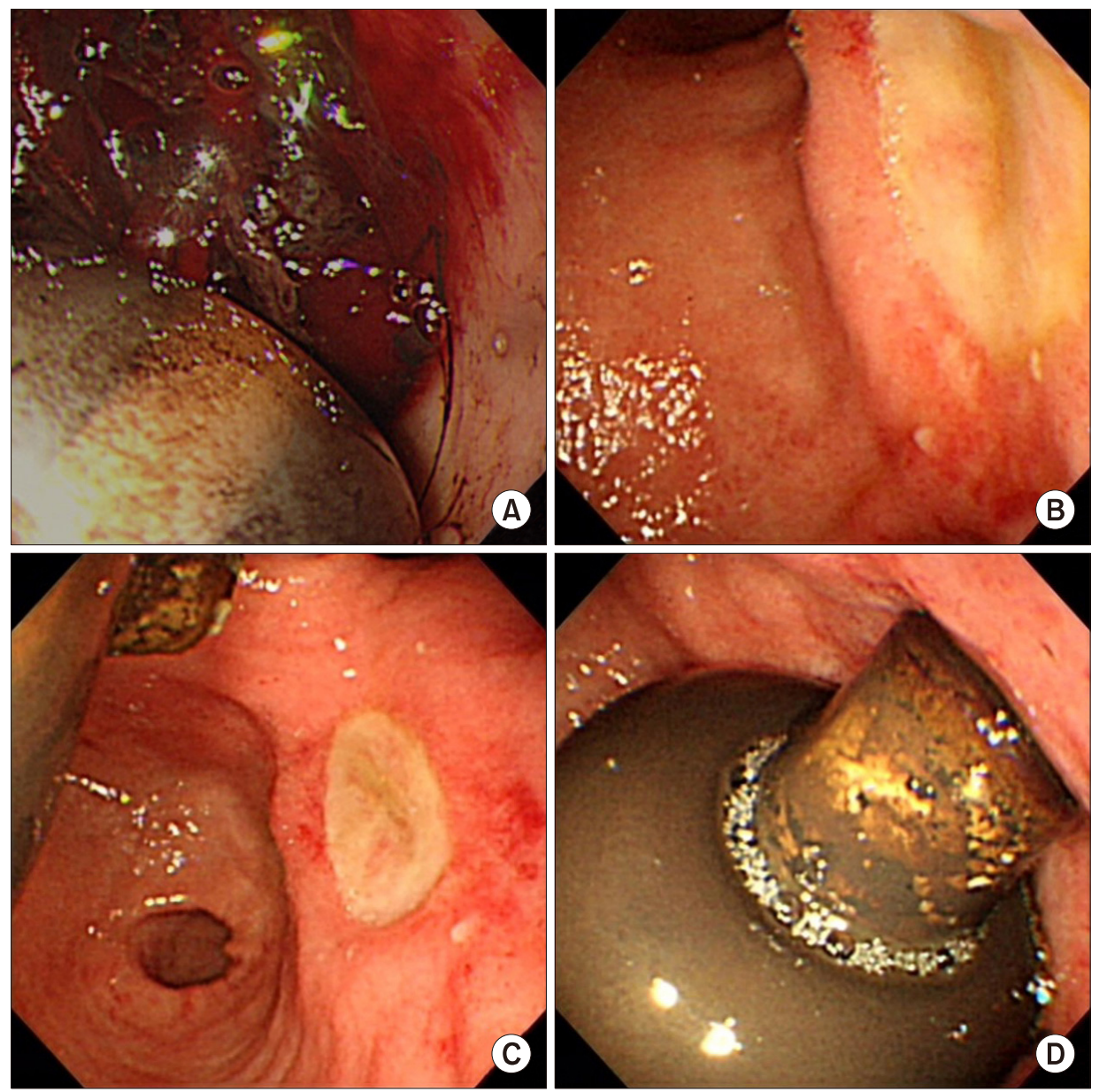

Fig. 1. (A) Emergency endoscopy reveals reddish blood clot near the tip of the balloon type tube. (B, C) Second-look endoscopy shows an active-staged ovoid ulcer at the posterior wall of the gastric antrum, the opposite site of the tube. (D) On airdeflation during endoscopy, the solid tip of the balloon type tube is in contact with the antral ulcer base.

Received: April 24, 2018 Revised: May 7, 2018 Accepted: May 7, 2018

Corresponding author: Kyung Ho Song

Department of Internal Medicine, Konyang University Hospital, 158 Gwanjeodong-ro, Seo-gu, Daejeon 35365, Korea

Tel: +82-42-600-9127, Fax: +82-42-600-9090, E-mail: postit@kyuh.ac.kr (1) ORCID: https://orcid.org/0000-0001-6661-3879

Copyright $\odot 2018$ Korean College of Helicobacter and Upper Gastrointestinal Research

() The Korean Journal of Helicobacter and Upper Gastrointestinal Research is an Open-Access Journal. All articles are distributed under the terms of the Creative Commons Attribution Non-Commercial License (http://creativecommons.org/licenses/by-nc/4.0) which permits unrestricted non-commercial use, distribution, and reproduction in any medium, provided the original work is properly cited. 
Percutaneous endoscopic gastrostomy (PEG) tube was replaced 2 months ago. Emergency endoscopy revealed a reddish blood clot near the tip of the balloon type tube (Fig. 1A). Second-look endoscopy revealed a clear, ovoid active-staged ulcer at the posterior wall of the gastric antrum (Fig. 1B, C). What other measures should be taken besides anti-ulcer drugs?

Answer: PEG insertion had been performed at the anterior wall of the gastric antrum, actually in other institute. When the stomach lumen was deflated during endoscopy, as shown in Fig. 1D, the solid tip of the balloon type tube was in contact with the antral ulcer base. In other words, it suggested that the rigid tip of the tube induced an ulcer via applying constant pressure to the opposite gastric wall. In order to prevent recurrence of bleeding, it was necessary to insert a new tube into the gastric body, or to replace it with a bell-type tube with a non-rigid tip. No recurrent bleeding episode have been reported over one year since the replacement with a bell-type tube.

PEG feeding is a good choice for maintaining nutritional intake in patients with swallowing difficulty due to neurological deficit. Endoscopists should be familiar with the complications of PEG and their preventive measures. ${ }^{1,2}$ The recommended site for insertion of the PEG tube is the anterior wall of the body, usually at the lower body. Even if the puncture site is determined, it should be confirmed by trans-illumination that it does not interfere with the transverse colon that can run on the anterior wall of the body. ${ }^{3}$ Occasionally, a novice may puncture on the antrum when inserting a PEG tube. Antrum is a rigid muscular part, and it has to play a role in pumping the digested food into the small intestine. If puncture is performed here, the antral pumping action can be disturbed. Besides, the risk of the pressure ulcer is higher because the lumen is much narrower than the gastric body. It should be noted that endoscopists should avoid puncturing the antrum during PEG tube insertion.

\section{REFERENCES}

1. Burney RE, Bryner BS. Safety and long-term outcomes of percutaneous endoscopic gastrostomy in patients with head and neck cancer. Surg Endosc 2015;29:3685-3689.

2. Sarkar P, Cole A, Scolding NJ, Rice CM. Percutaneous endoscopic gastrostomy tube insertion in neurodegenerative disease: a retrospective study and literature review. Clin Endosc 2017;50:270-278.

3. Pitsinis V, Roberts P. Gastrocolic fistula as a complication of percutaneous endoscopic gastrostomy. Eur J Clin Nutr 2003; 57:876-878. 